\title{
TIPE2 ameliorates lipopolysaccharide-induced apoptosis and inflammation in acute lung injury
}

\author{
Xiaojing $\mathrm{Wu}^{1} \cdot$ Qian Kong ${ }^{1} \cdot$ Liying Zhan $^{1} \cdot$ Zhen Qiu $^{1} \cdot$ Qin Huang $^{1} \cdot$ Xuemin Song $^{2}$
}

Received: 4 May 2019 / Revised: 25 August 2019 / Accepted: 29 August 2019 / Published online: 5 September 2019

(c) Springer Nature Switzerland AG 2019

\begin{abstract}
Objective Tumour necrosis factor- $\alpha$-induced protein 8 -like 2 (TIPE2) has strong anti-inflammatory properties. However, it is unknown whether increased TIPE2 is protective against lipopolysaccharide (LPS)-induced ALI. In the current study, we aimed to investigate whether increased TIPE2 can exert protective effects in a mouse model of ALI induced by LPS.

Methods We administered TIPE2 adeno-associated virus (AAV-TIPE2) intratracheally into the lungs of mice. Three weeks later, ALI was induced by intratracheal injection of LPS into BALB/c mice. Twenty-four hours later, lung bronchoalveolar lavage fluid (BALF) was acquired to analyse cells and protein, arterial blood was collected for arterial blood gas analysis and the determination of pro-inflammatory factor levels, and lung issues were collected for histologic examination, transmission electron microscopy (TEM), TUNEL staining, wet/dry (W/D) weight ratio analysis, myeloperoxidase (MPO) activity analysis and blot analysis of protein expression.

Results We found that TIPE2 overexpression markedly mitigated LPS-induced lung injury, which was evaluated by the deterioration of histopathology, histologic scores, the W/D weight ratio, and total protein expression in the BALF. Moreover, TIPE2 overexpression markedly attenuated lung inflammation, as evidenced by the downregulation of polymorphonuclear neutrophils (PMNs) in the BALF, lung MPO activity, and pro-inflammatory cytokine levels in the serum. Moreover, TIPE2 overexpression not only dramatically prevented LPS-induced pulmonary cell apoptosis in mice but also blocked LPS-activated JNK phosphorylation and NF-kB p65 nuclear translocation.

Conclusions Our study shows that the increased expression of AAV-mediated TIPE2 in the lungs of mice inhibits acute inflammation and apoptosis and suppresses the activation of NF-kB and JNK in a murine model of ALI.
\end{abstract}

Keywords Acute lung injury $\cdot$ TIPE2 $\cdot$ Apoptosis $\cdot$ Inflammation $\cdot$ Cytokines $\cdot$ NF-kB $\cdot$ JNK

$\begin{array}{ll}\text { Abbreviations } \\ \text { ALI } & \text { Acute lung injury } \\ \text { ARDS } & \text { Acute respiratory distress syndrome } \\ \text { BALF } & \text { Bronchoalveolar lavage fluid } \\ \text { ELISA } & \text { Enzyme-linked immunosorbent assay } \\ \text { i.t. } & \text { Intratracheal } \\ \text { LPS } & \text { Lipopolysaccharide } \\ \text { MPO } & \text { Myeloperoxidase }\end{array}$

Responsible Editor: John Di Battista.

Xuemin Song

sxmc11018@163.com

1 Department of Anesthesiology, Renmin Hospital of Wuhan University, Wuhan, Hubei Province, China

2 Department of Anesthesiology and Critical Care Medicine, Zhongnan Hospital of Wuhan University, Wuhan, Hubei Province, China
PBS Phosphate-buffered saline

PMNs Polymorphonuclear neutrophils

TUNEL Deoxynucleotidyl transferase dUTP nick end labeling

W/D Wet-to-dry

TIPE2 Tumor necrosis factor- $\alpha$-induced protein 8 -like 2

\section{Introduction}

Acute lung injury (ALI) and acute respiratory distress syndrome (ARDS) are life-threatening medical conditions with high morbidity and mortality rates, and they are triggered by common pathologies such as sepsis, trauma and pneumonia [1]. ALI pathophysiology is characterised by the increased permeability of the alveolar-capillary barrier, interstitial edema, neutrophil recruitment, and inflammatory 
stress-induced cell apoptosis [2, 3]. The pathophysiology of sepsis-induced ALI is characterised by complex mechanisms that involve cell inflammation, cytokine production, and abnormal apoptosis [4]. To date, there is no effective pharmacological approach to treat ALI.

Apoptosis, known as programmed cell death, is essential for the selective elimination of cells. However, the dysregulation of apoptosis pathways has been demonstrated to contribute to epithelial and endothelial injury, which are characteristic of ALI [5]. The inhibition of apoptosis increases the animal survival rate in an LPS-induced ALI model [6].

Lipopolysaccharide (LPS), a main component of the outer membrane of gram-negative bacteria, is widely used to induce animal models of ALI through intratracheal instillation [7]. After binding with Toll-like receptor 4 (TLR4), LPS induces the activation of downstream signalling pathways responsible for the infiltration of inflammatory cells (i.e., neutrophils) into the lungs and the production of proinflammatory cytokines [8]. The binding of LPS to TLR4 also induces IкB- $\alpha$ phosphorylation and degradation, promotes the nuclear translocation and activation of NF- $\mathrm{\kappa B}$, and subsequently leads to the excessive release of pro-inflammatory cytokines [e.g., tumour necrosis factor- $\alpha$ (TNF- $\alpha$ ), interleukin (IL)-1 $\beta$, IL-6] [9]. Moreover, LPS can activate JNK, a member of the MAPK family. Activated JNK can phosphorylate numerous mitochondrial proteins, including Bcl-2 and Bcl-xl [10, 11]. Subsequently, cytochrome $\mathrm{c}$ is released from the mitochondria, which leads to the activation of death signals [12]. JNK activation is essential for LPS-induced macrophage apoptosis during sepsis [13].

Tumour necrosis factor- $\alpha$-induced protein-8 (TNFAIP8)like 2 (TIPE2), which is an essential negative regulator of TLR and TCR function, has been confirmed to inhibit caspase-mediated apoptosis [14]. TIPE2 has been reported to be a negative regulator of the activating protein (AP)-1, NF- $\kappa B$, JNK, and p38 pathways [15].

Whether TIPE2 has a therapeutic effect on LPS-induced ALI has not been reported. The current study was designed to test the hypothesis that TIPE2 attenuates LPS-induced ALI through the inhibition of lung inflammation and apoptosis, which may be associated with suppressing NF- $\mathrm{\kappa B}$ and JNK activation.

\section{Materials and methods}

\section{Reagents}

LPS (E. coli 0111:B4) was obtained from Sigma-Aldrich (St. Louis, MO). Rabbit polyclonal Bax, caspase 3, TIPE2 and Lamin A antibody were sourced from Abcam limited. Rabbit polyclonal JNK, rabbit monoclonal p-JNK and $\beta$-actin antibody were from Cell Signaling Technology (Boston, MA).
Rabbit polyclonal Bcl-2, NF- $\mathrm{KB}$ p65, and caspase 9 antibodies were from ProteintechGroup, Inc (Wuhan, China). HRPconjugated secondary antibody was obtained from Boster Biological Technology co. LTD (Wuhan, China). Enzymelinked immunosorbent assay (ELISA) kits were purchased from Abclonal Technology (USA). MPO kit was obtained from Nanjing Biohelper Co., Ltd. (Nanjing, China).

\section{Animals}

Adult male BABL/c mice (6-8 weeks) weighing 20-25 g were purchased from the Wuhan Institute of Biological Products Co., Ltd. (Wuhan, China). The mice were housed under specific pathogen-free (SPF) conditions that provide relative humidity ranging between $55 \%$ and $65 \%$, temperature of $22 \pm 2{ }^{\circ} \mathrm{C}$, a $12: 12 \mathrm{~h}$ light-dark cycle, with free access to food and water. The animals were adapted to this environment for 1 week before the experiment. This study was approved by Medical Ethics Committee of Renmin Hospital of Wuhan University and was performed in accordance with the National Institutes of Health Guidelines for the Care and Use of Laboratory Animals.

\section{Adenovirus gene delivery}

A recombinant adeno-associated virus containing the mouse TIPE2 gene was purchased from Hanheng Company (Hanheng Biotechnology Co., Ltd., Shanghai, China). An adeno-associated virus expressing no transgene was used as a negative control (pAAV-IRES-ZsGreen). Twenty-one days before LPS instillation, BALB/c mice were anaesthetized using sodium pentobarbital and given $5 \times 10^{10}$ vector genomes $(\mathrm{vg})$ of rAAV6-FLAG-mTIPE2 $\left(5 \times 10^{12} \mathrm{vg} / \mathrm{ml}\right)$ in $50 \mu \mathrm{l}$ of PBS via intratracheal (i.t.) administration to induce the overexpression of pulmonary TIPE2. Control mice were treated with the control adeno-associated virus. The efficacy of the fusion protein was evaluated by western blotting.

\section{Experimental design and animal models of ALI}

Forty mice were randomly divided into four groups ( $n=10$ per group): the control group (PBS); the LPS group (LPS); the TIPE2 + PBS group (AAV-TIPE2 + PBS); and the TIPE2 + LPS group (AAV-TIPE2 + LPS). As previously described by Matute-Bello et al. [18], to establish the ALI model, mice were anaesthetized with an intraperitoneal (i.p.) injection of pentobarbital sodium $(50 \mathrm{mg} / \mathrm{kg})$, orally intubated with a sterile plastic catheter and subsequently intratracheally injected with LPS (Escherichia coli 055: B5; Sigma, St. Louis, MO, USA) at a dose of $5 \mathrm{mg} / \mathrm{kg}$ body weight. Control mice were intratracheally administered $50 \mu$ of sterile phosphatebuffered saline (PBS). Twenty-four hours after LPS treatment, the mice were sacrificed by an i.p. injection of pentobarbital 
(50 mg/kg; Sigma). Arterial blood was collected, and then a median sternotomy was performed to expose the lungs. In each mouse, after the hilum of the right lung was ligated, the left lung was lavaged to obtain the BALF. The right upper lobe of the lung was excised to calculate the lung wet/dry weight ratio. Lung tissues from part of the right middle lobe of the lung were taken for HE staining, immunohistochemical staining, transmission electron microscopy, and TUNEL staining. Lung tissues from part of the right lower lobe of lung were taken for MPO activity detection and western blotting. Lung tissues were snap-frozen in liquid nitrogen and stored at $-80^{\circ} \mathrm{C}$ for later analysis.

\section{Histopathological lung examination}

Lung tissues were harvested for observing morphologic alterations at $24 \mathrm{~h}$ after LPS or PBS administration. The right middle lobe of lung were excised, washed and fixed with $4 \%(\mathrm{v} / \mathrm{v})$ paraformaldehyde for $24 \mathrm{~h}$ at $4{ }^{\circ} \mathrm{C}$. Lung tissues were embedded in paraffin, sectioned at $4 \mu \mathrm{m}$ thickness, dewaxed and rehydrated, and stained with hematoxylin and eosin (H\&E) solution (hematoxylin, MHS16; eosin, HT110132; Sigma-Aldrich, USA) to estimate inflammation in alveolar and peribronchial lesions. The stained slides were then observed with the light microscope and the digital micrographs were taken for analyzing. Histologic changes were evaluated by a pathologist blinded to the experiment. The degree of lung injury was graded using a histologic ALI scoring system based on histologic features, including neutrophils infiltration, hyaling membranes, proteinaceous debris, and alveolar septal thickening [16].

\section{Immunohistochemistry stain}

Sections of paraffin-embedded tissue were subjected to immunohistochemical staining. The Rabbit polyclonal TIPE2 antibody (15940-1-AP, Proteintech Group, Inc. USA) was used at 1:250. Sections were incubated with primary antibody $\left(4^{\circ} \mathrm{C}, 15 \mathrm{~h}\right)$, followed with a poly-horseradish peroxidase anti-rabbit secondary antibody (dilution ratio 1:10,000; A24531, Thermo Fisher Scientific Inc. USA) incubated $\left(37^{\circ} \mathrm{C}, 20 \mathrm{~min}\right)$, and diaminobenzidine (DAB) was used to visualize the complex. Subsequently, the sections were counterstained with hematoxylin, dehydrated, and mounted. The expression of TIPE2 was evaluated using a light microscope (BX51; Olympus Corporation, Tokyo, Japan).

\section{Ultrastructural changes of lung tissues by transmission electron microscopy}

The lungs were isolated and cut into $1-2-\mathrm{mm}^{3}$ cubes. Lung tissue samples were fixed by immersion in $2.5 \%$ glutaraldehyde buffer for $24 \mathrm{~h}$ at $4{ }^{\circ} \mathrm{C}$, washed with PBS solution three times, post-fixed for $1 \mathrm{~h}$ in $1 \%$ osmium tetroxide, dehydrated in graded solutions of ethyl alcohol (30\%, 50\%, 70\%, 90\% and 100\%), and embedded in epoxy resin. Ultrathin sections $(70 \mathrm{~nm})$ that were double-stained with uranyl acetate and lead citrate were examined under a transmission electron microscope (Hitachi H-600, Hitachi, Tokyo, Japan).

\section{TdT-mediated dUTP nick end labelling (TUNEL) staining}

Apoptosis was detected and quantified by the TUNEL assay using the In Situ Cell Death Detection Kit (Roche Diagnostics GmbH, Mannheim, Germany.) according to the manufacturer's protocol. Apoptotic cells exhibited brownish staining in the cell nuclei. Ten random sections of the lung from each mouse were analysed without knowledge of the group of mice from which the lung tissue was taken, and the apoptosis index was expressed as a percentage of TUNEL-positive cells. The examination was performed by two pathologists blinded to the experimental design.

\section{Inflammatory cell counting and protein concentration determination in the BALF}

To obtain the BALF, the lungs were lavaged three times with ice-cold PBS $(0.5 \mathrm{ml})$ and withdrawn each time using a tracheal cannula (a total volume of $1.5 \mathrm{ml}$ ). The collected BALF was centrifuged at $1000 \times g$ for $15 \mathrm{~min}$ at $4{ }^{\circ} \mathrm{C}$, and the supernatants were collected and frozen at $-80^{\circ} \mathrm{C}$ for subsequent assays. The cell pellet was resuspended in PBS, and after excluding the dead cells by trypan blue staining, the total number of inflammatory cells in the BALF was determined by counting the cells with a haemocytometer (Beckman Coulter, Inc). To analyse the cell numbers, $100 \mu \mathrm{l}$ of BALF was centrifuged onto slides by a Cytospin (Thermo Fisher Scientific, Waltham, USA). After the slides were dried, the cells were fixed and stained using Wright Stain solution (32857, Sigma, USA) according to the manufacturer's instructions. The number of polymorphonuclear neutrophils (PMNs) was classified by a laboratory technologist blinded to the experimental design to determine the percentage of neutrophils. The frozen BALF supernatant was thawed and thoroughly mixed, and the total protein concentration was determined by the BCA (bicinchoninic acid) method.

\section{Arterial blood gas analysis}

After mice were anesthetized, the arterial blood sample was collected with a heparinized syringe from the carotid artery. The arterial blood samples were immediately injected into 
an ABL700 Radiometer (Radiometer America, USA) to measure $\mathrm{pH}$ value, partial gas pressures of oxygen $\left(\mathrm{PaO}_{2}\right)$, $\mathrm{PaO}_{2} /$ fraction of inspired oxygen $\left(\mathrm{FiO}_{2}\right)$, and carbon dioxide $\left(\mathrm{PaCO}_{2}\right)$.

\section{Lung wet/dry weight ratio}

The magnitude of pulmonary edema was determined by calculating the lung wet/dry weight ratio. The right upper lobe of the lung was excised, washed with phosphate-buffered saline (PBS), blotted and then weighed to obtain the "wet" weight. The lung was then placed in an oven for $48 \mathrm{~h}$ at $65^{\circ} \mathrm{C}$ and weighed to obtain the "dry" weight. The wet/dry ratio was calculated to quantify the degree of pulmonary edema.

\section{MPO activity assay}

RIPA lysis buffer was used for lysing the lung tissues, and $10 \mathrm{mg}$ of tissue was used for each test sample. After washing with cold PBS, the tissues were resuspended in four volumes of MPO assay buffer and then centrifuged $(13,000 \times g$ for $10 \mathrm{~min}, 4^{\circ} \mathrm{C}$ ). The supernatant was collected and transferred to clean tubes, which were placed on ice. The MPO activity was assayed using a Myeloperoxidase Activity Assay Kit (Abcam, ab105136) by measuring the absorbance of the sample at $460 \mathrm{~nm}$ using a microplate reader (Bio-Rad Laboratories, Hercules, CA, USA). The specific MPO activity in the lungs is expressed as unit/mg protein.

\section{Measurement of pro-inflammatory cytokines in serum}

Blood was collected from the carotid artery, and serum was obtained following centrifugation $(1000 \times g$ for $10 \mathrm{~min})$. The levels of TNF- $\alpha$, IL- 6 and IL- $1 \beta$ in serum were determined using enzyme-linked immunosorbent assay (ELISA) kits according to the manufacturer's instructions (R\&D Systems, Minneapolis, MN, USA). The absorbance was measured at $450 \mathrm{~nm}$ using an ELISA reader (BioTek Instruments, Inc., USA).

\section{Western blot analysis}

$24 \mathrm{~h}$ after the injection of LPS, the lung tissues were harvested and snap-frozen in liquid nitrogen until homogenization. The lung tissues were homogenized using a homogenizer with tissue nuclear and cytoplasmic extraction reagents (Sigma-Aldrich, USA), according to the manufacturer's instructions. Protein concentrations were determined using the BCA protein assay kit (Invitrogen; Thermo Scientific). Equal amounts of protein $(50 \mu \mathrm{g})$ were loaded per well on sodium dodecyl sulphate-polyacrylamide gel electrophoresis
(SDS-PAGE) and transferred onto polyvinylidene difluoride membranes. The resulting membranes were blocked by incubation with 5\% skim milk in TBST at room temperature for $2 \mathrm{~h}$ on a rotary shaker, followed by washing with TBST. Subsequently, the membranes were incubated with specific primary antibody overnight at $4{ }^{\circ} \mathrm{C}$. The membranes were washed with TBST followed by incubation with horseradish peroxidase (HRP)-conjugated secondary antibody at room temperature for $1 \mathrm{~h}$. The blots were washed TBST and detected using an enhanced chemiluminescence (ECL) western blotting detection kit. The proteins bands were observed using an ECL western blotting analysis system (Bio-Rad Laboratories, Inc., USA) and quantified by densitometry (Image Lab software version 5.2.1).

\section{Statistical analysis}

The data are expressed as the mean \pm SEM. The statistical analysis was performed using GraphPad Prism (version 7.0; GraphPad Software, Inc., La Jolla, CA, USA) by one-way analysis of variance (ANOVA) followed by Dunnett's least significant difference post hoc test. $P<0.05$ was considered statistically significant.

\section{Results}

\section{TIPE2 overexpression attenuates LPS-induced lung injury in mice}

In the present study, we first investigated the effects of TIPE2 overexpression on lung histopathology and function in mice challenged with LPS (Fig. 1). The lung tissues were harvested $24 \mathrm{~h}$ after LPS stimulation and subjected to H\&E staining. There were no obvious histological changes in the lung tissues of the mice group A and group C. Significant pathological changes, including pulmonary capillary congestion, pulmonary interstitial edema, mass inflammatory cell infiltration into the alveolar space and lung interstitium, and alveolar wall thickening, were observed in the lung tissues of LPS-challenged mice. A subsequent western blot assay further demonstrated that TIPE2 protein levels were significantly increased in the lung tissues of AAV-TIPE2infected mice. Treatment with AAV-TIPE2 significantly attenuated the histopathological changes induced by LPS (Fig. 1a). In addition, a scoring system was used to assess the degree of lung injury. As shown in Fig. 1b, the quantitative scoring of histological lung injury in the ALI mice was markedly increased compared with that in the control group $24 \mathrm{~h}$ after LPS challenge. However, recombinant adenoassociated virus-mediated TIPE2 overexpression markedly decreased the pathological scores compared with those of the LPS group. 


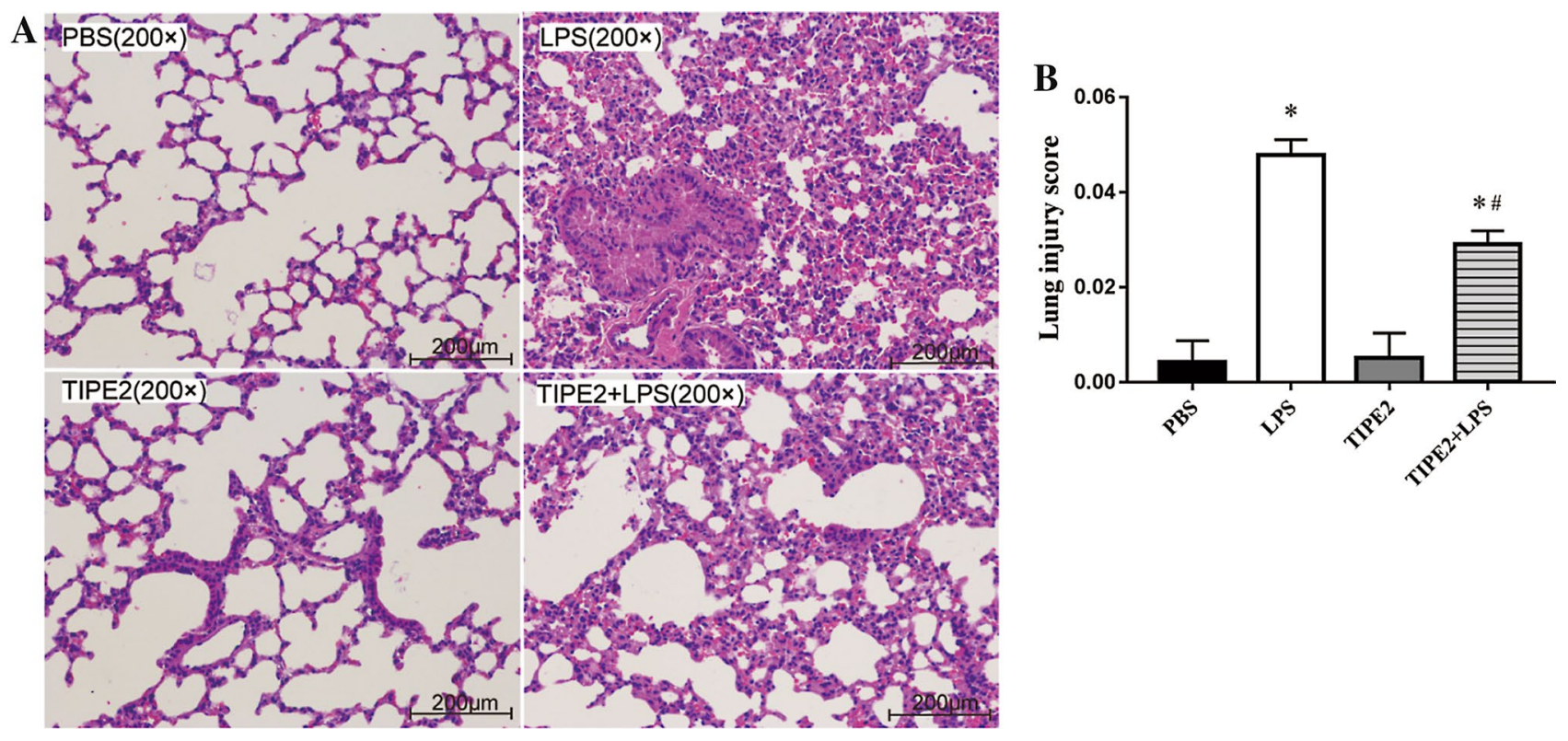

Fig. 1 TIPE2 overexpression attenuates the lung histopathological changes in LPS-challenged mice. Acute lung injury was induced by i.t. administration of LPS at a dose of $5 \mathrm{mg} / \mathrm{kg}$ body weight. Control mice were intratracheally administered $50 \mu \mathrm{l}$ of sterile PBS. $24 \mathrm{~h}$ after the LPS/PBS treatment, the mice were sacrificed by an i.p. injection of pentobarbital, the lung tissues without lavage were collected

\section{TIPE2 overexpression attenuates ultrastructural changes in the lung tissues of LPS-challenged mice}

Transmission electron microscopy was used to examine the ultrastructural changes of lung tissues (Fig. 2). In the control group, there were abundant mitochondria with regularly arranged mitochondrial cristae and homogeneous matrix. Lungs from LPS-treated mice exhibited a disordered arrangement of mitochondrial cristae, and the number of lamellar bodies was decreased. In the TIPE2 + LPS group, the pathologic damage was significantly alleviated compared with that in the LPS group.

\section{TIPE2 overexpression inhibits pulmonary cell apoptosis in LPS-challenged mice}

We investigated the effects of TIPE2 overexpression on lung cell apoptosis in LPS-challenged mice by TUNEL staining. TUNEL staining revealed few apoptotic cells in the lungs of the control group. After the administration of intratracheal LPS, unlike in control mice, numerous lung cells were strongly positive for TUNEL staining. However, in the lung tissues of AAV-TIPE2-treated mice, a few of the lung cells were TUNEL-positive (Fig. 3a). For quantitative measurement, the percentage of TUNEL-positive lung cells was analysed for each specimen (Fig. 3b). The results showed that LPS-challenged mice showed a for measuring the histopathology, histologic injury scores and ultrastructural changes of the lung. a Representative histology sections of lung tissues under light microscope (H\&E staining, magnification, $\times 200$ ); b Lung histologic injury score. The data are presented as mean \pm SEM. $n=10$ /group, ${ }^{*} P<0.05$ versus PBS group; ${ }^{\#} P<0.05$ versus LPS group

significant increase in the number of apoptotic cells, which was reduced by AAV-TIPE2 treatment. These data indicated that TIPE2 overexpression inhibited apoptosis in the lung after LPS challenge.

\section{TIPE2 overexpression reduces pulmonary vascular permeability and neutrophil infiltration into the lungs during $A L I$ induced by LPS}

The lung W/D ratio and BALF protein concentration are two commonly used indicators of pulmonary vascular permeability, which is an important characteristic of ALI/ARDS. LPS-challenged mice showed a significant increase in the lung W/D ratio (Fig. 4a) and BALF protein concentration (Fig. 4b) when compared with those of the control group, and these levels were decreased by AAV-TIPE2 treatment. We also detected the ratio of the number of PMNs relative to the number of total cells in the BALF and the activity of MPO, an indicator of neutrophil infiltration, in the lung $24 \mathrm{~h}$ after LPS administration. Compared with those in the control group, the PMN/total cell ratio in the BALF (Fig. 4c) and lung MPO activity (Fig. 4d) in LPS-challenged mice were dramatically increased, and these levels were inhibited by AAV-TIPE2 treatment. These results suggest that TIPE2 overexpression attenuates lung edema and inflammation in LPS-challenged mice. 
Fig. 2 TIPE2 overexpression attenuates the lung ultrastructural changes in LPS-challenged mice. Ultrastructural changes of lung tissues under transmission electron microscope $(\times 10,000)$. $\mathrm{Lb}$ lamellar body, $\mathrm{Nu}$ nucleus, $M$ mitochondria
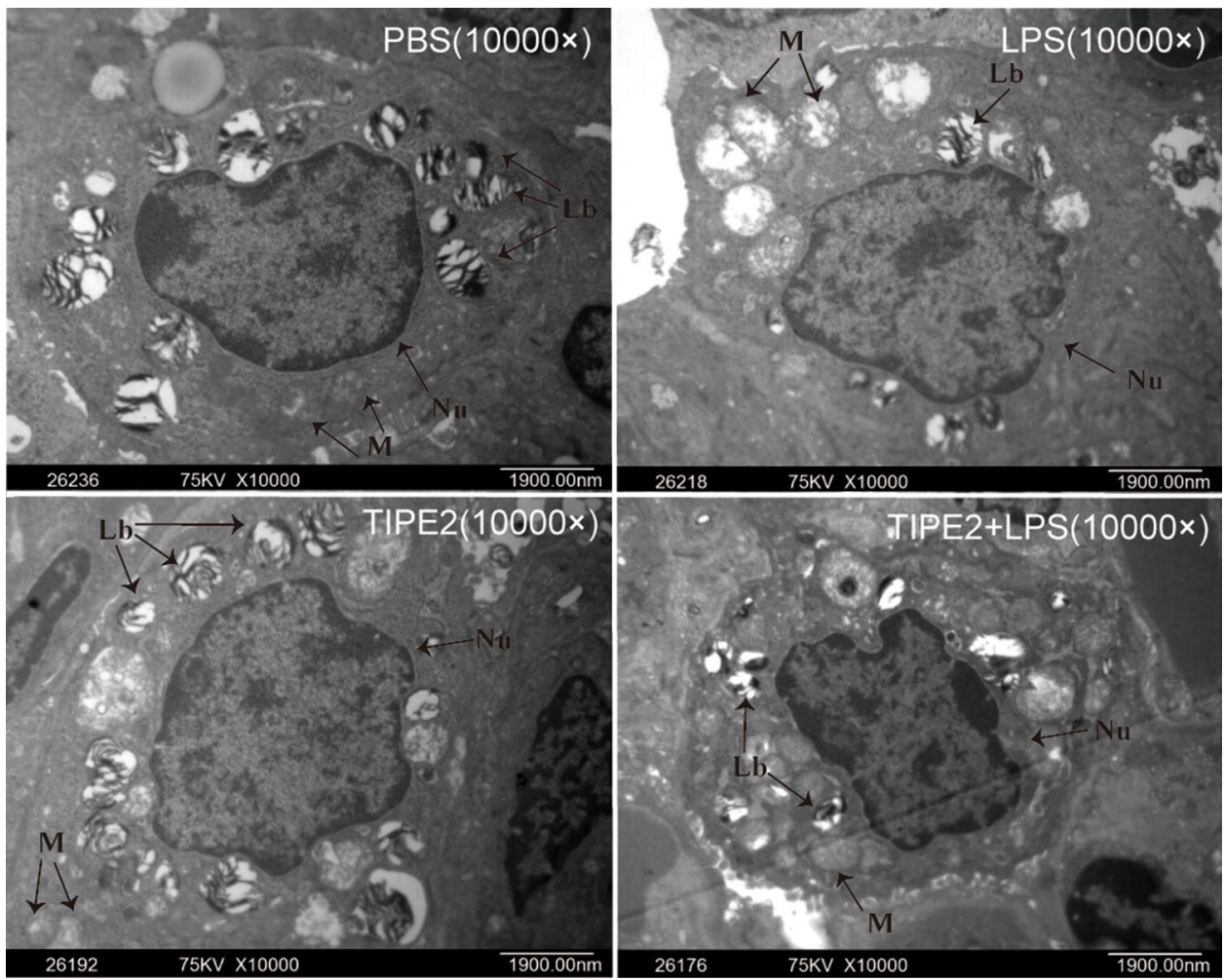
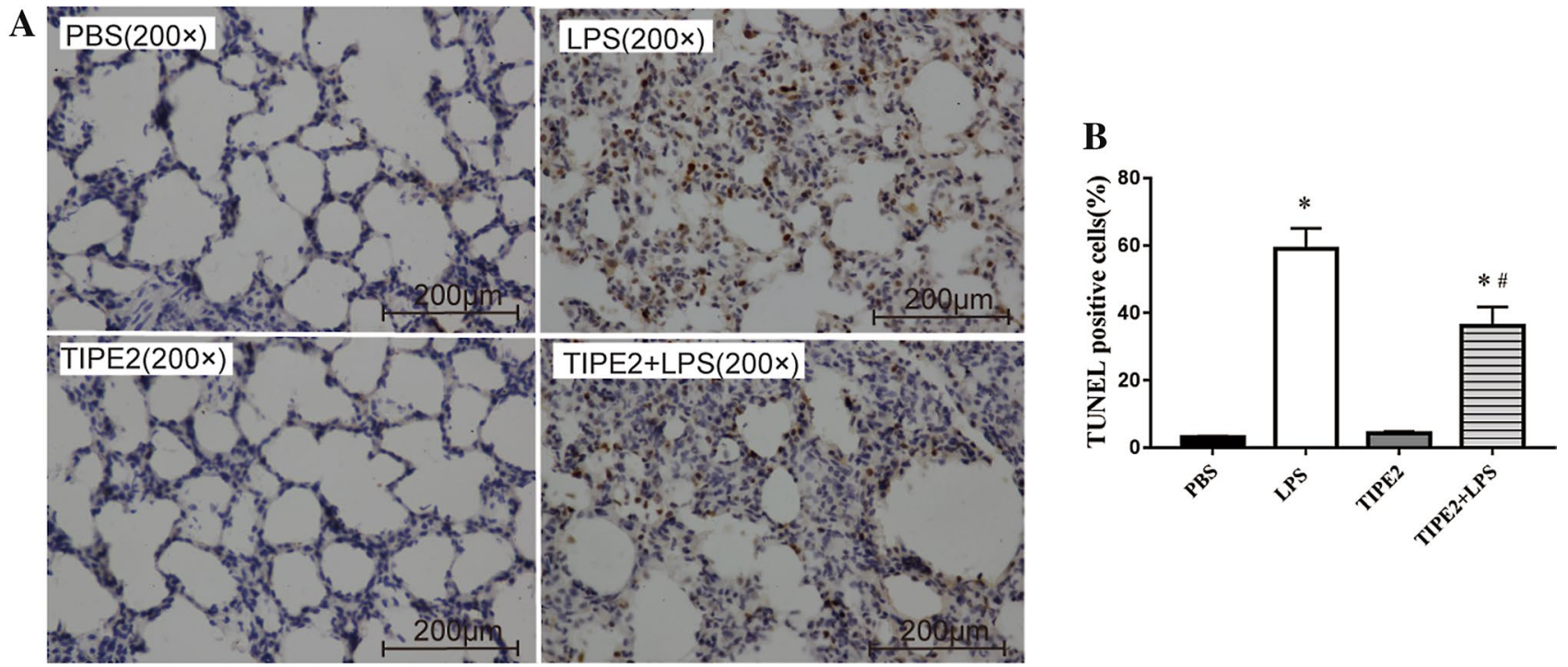

Fig. 3 TIPE2 overexpression prevented the lung cell apoptosis in LPS-challenged mice. The mice were treated as described in Fig. 1. The lung samples were collected for measuring TUNEL staining at $24 \mathrm{~h}$ after PBS/LPS administration. a Representative lung TUNEL

\section{TIPE2 overexpression improves pulmonary dysfunction during ALI induced by LPS}

As shown in Fig. 5, the arterial blood gas analysis of mice that received LPS treatment showed significant staining $(\times 400)$; b Percentage of TUNEL-positive cells. The data are presented as mean \pm SEM. $n=10 /$ group, ${ }^{*} P<0.05$ versus $P B S$ group; ${ }^{\#} P<0.05$ versus LPS group

changes compared with that of the control group, with the $\mathrm{pH}$ (Fig. 5a), partial pressure of arterial oxygen $\left(\mathrm{PaO}_{2}\right)$ (Fig. 5b) and $\mathrm{PaO}_{2} / \mathrm{FiO}_{2}$ (Fig. 5c) decreasing and the partial pressure of arterial carbon dioxide $\left(\mathrm{PaCO}_{2}\right)$ (Fig. 5d) increasing. The $\mathrm{PaO}_{2} / \mathrm{FiO}_{2}$ of the mice in the LPS group 
Fig. 4 TIPE2 overexpression reduced Lung W/D ratio, BALF protein concentration, PMNs/ total cells in BALF as well as lung MPO activity in LPSchallenged mice. The mice were treated as described in Fig. 1. BALF and lung tissues were collected at $24 \mathrm{~h}$ after LPS or PBS administration. a Lung W/D ratio; b BALF protein concentration; c PMNs/total cells in BALF; d lung MPO activity. The data are presented as mean \pm SEM. $n=10 /$ group, ${ }^{*} P<0.05$ versus PBS group; ${ }^{\#} P<0.05$ versus LPS group
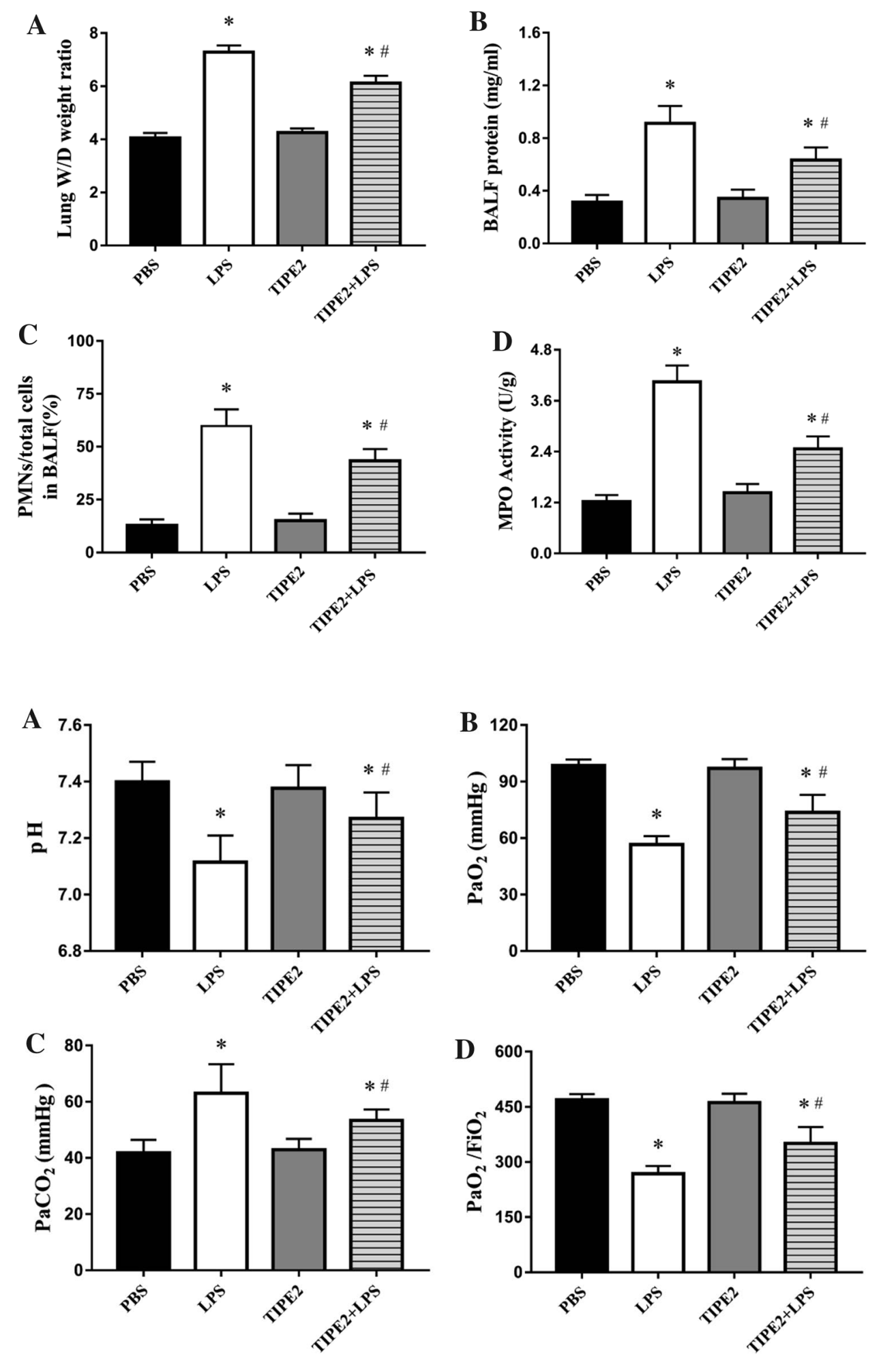

Fig. 5 TIPE2 overexpression improved pulmonary dysfunction during ALI induced by LPS. The mice were treated as described in Fig. 1. Arterial blood was collected for blood gas analysis at $24 \mathrm{~h}$ after LPS or PBS administration. $\mathbf{a} \mathrm{pH} ; \mathbf{b}$ $\mathrm{PaO}_{2} ; \mathbf{c ~} \mathrm{PaO}_{2} / \mathrm{FiO}_{2} ; \mathbf{d} \mathrm{PaCO}_{2}$. The data are presented as mean \pm SEM. $n=10$ /group, ${ }^{*} P<0.05$ versus $P B S$ group; ${ }^{\#} P<0.05$ versus LPS group achieved clinical diagnostic criteria for ALI $(<300)$. However, TIPE2 overexpression effectively mitigated the change in arterial oxygenation. Compared with those in the LPS group, the $\mathrm{pH}$ and $\mathrm{PaO}_{2}$ were increased, and $\mathrm{PaCO}_{2}$ was decreased in the TIPE2 + LPS group. The $\mathrm{PaO}_{2} / \mathrm{FiO}_{2}$ in the TIPE2 + LPS group recovered to normal levels and was higher than that in the LPS group. 


\section{TIPE2 overexpression downregulates the cytokines in the serum of LPS-challenged mice}

As depicted in Fig. 6, we found that the levels of the proinflammatory cytokines TNF- $\alpha$ (Fig. 6a), IL-6 (Fig. 6b) and IL-1 $\beta$ (Fig. 6c) in the serum were significantly increased $24 \mathrm{~h}$ after LPS challenge in mice. AAV-TIPE2 treatment significantly downregulated the levels of pro-inflammatory cytokines in the serum of LPS-challenged mice.

\section{TIPE2 overexpression suppresses the activation of JNK and NF-KB as well as the protein expression of genes involved in apoptosis and lung injury induced by LPS}

Previous studies have shown that TIPE2 is a negative regulator of the NF- $\mathrm{kB}$, JNK, and p38 MAPK pathways in macrophages [15]. The effect of TIPE2 on NF- $\mathrm{BB}$ and JNK activation was assessed in mice after LPS challenge. To evaluate the effect of TIPE2 overexpression on lung cell apoptosis in LPS-challenged mice, the protein expression levels of anti-apoptotic proteins (Bcl-2) and proapoptotic proteins (Bax, cleaved caspase-3, cleaved caspase-9) in the lung tissues were analysed by western blotting. As shown in Fig. 7, compared with control mice, mice with LPS-induced ALI exhibited decreased Bcl-2 expression and increased Bax, Bax/Bcl-2, cleaved caspase-3, and cleaved caspase-9 protein expression in lung tissue samples, whereas the changes in the expression of the proteins were reversed by AAV-TIPE2 treatment. In addition, LPS stimulation markedly increased nuclear NF-KB p65 and phosphorylated JNK (p-JNK) expression and decreased TIPE2 expression in the lungs compared with that in the control group. However, adeno-associated virus-mediated TIPE2 overexpression suppressed the increase in the expression of nuclear NF- $\mathrm{BB}$ p65 and $\mathrm{p}-\mathrm{JNK}$ induced by LPS.

\section{Expression of TIPE2 protein in the lung}

The expression of the TIPE2 protein was significantly increased in the alveolar epithelium after AAV-TIPE2 administration (Fig. 8). The expression of TIPE2 was decreased $24 \mathrm{~h}$ after LPS challenge. The TIPE2 + LPS group exhibited higher expression of TIPE2 compared with that in the LPS group.

\section{Discussion}

ALI and ARDS are the two main causes of acute lung failure, which is characterised by high morbidity and mortality and for which effective therapeutic strategies are lacking [17]. Thus, identifying novel therapeutic treatments for ALI is urgently needed. In the current study, we found that TIPE2 overexpression attenuated LPS-induced ALI in mice via its anti-inflammatory and anti-apoptotic effects. First, TIPE2 overexpression significantly improved LPS-induced lung injury, as evidenced by changes in histopathology, the lung W/D weight ratio, BALF protein concentration, and arterial blood gas. Second, TIPE2 overexpression decreased inflammatory cell infiltration into the lungs and pulmonary cell apoptosis. Third, the levels of pro-inflammatory cytokines in the serum of LPS-challenged mice were reduced by AAVTIPE2 treatment. Fourth, TIPE2 overexpression inhibited LPS-induced NF- $\kappa B$ p 65 nuclear translocation and JNK phosphorylation in the lungs. Finally, TIPE2 overexpression prevented lung cell apoptosis by downregulating the expression of pro-apoptotic proteins (Bax, cleaved caspase-3, and cleaved caspase-8) and upregulating the expression of an anti-apoptotic protein (Bcl-2) in the lungs of LPS-challenged mice. In conclusion, these results demonstrate that TIPE2 overexpression ameliorates LPS-induced ALI via reducing
A

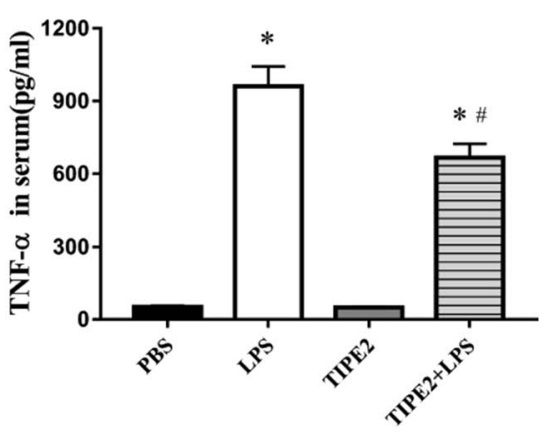

Fig. 6 TIPE2 overexpression reduced LPS-induced proinflammatory cytokine levels in mice. The mice were treated as described in Fig. 1. Blood sample was collected for measuring the pro-inflammatory
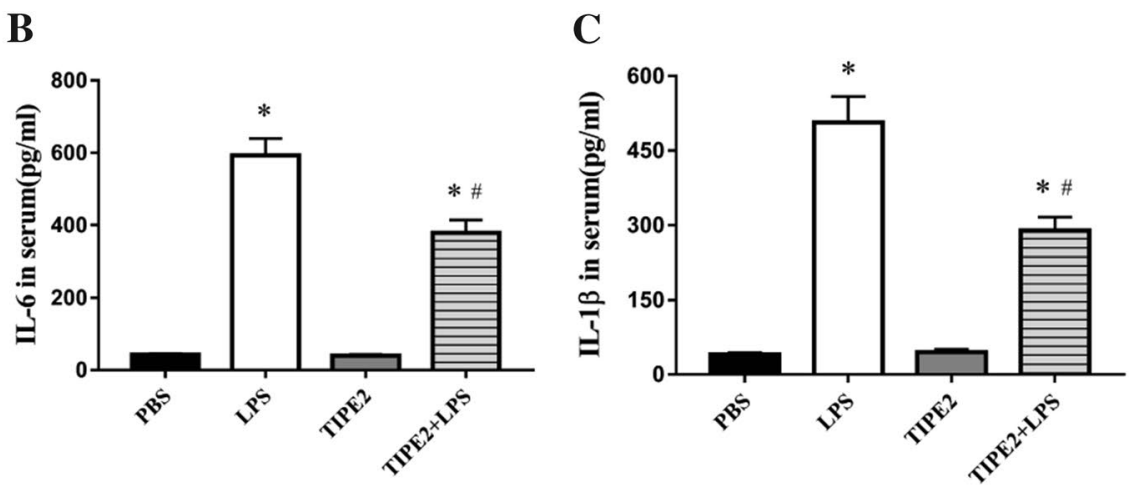

cytokines levels at $24 \mathrm{~h}$ after LPS or PBS administration. a TNF- $\alpha$; b IL-6; c IL-1 $\beta$. The data are presented as mean \pm SEM. $n=10 /$ group, ${ }^{*} P<0.05$ versus PBS group; ${ }^{\#} P<0.05$ versus LPS group 
A $\quad \frac{\text { WT }}{\text { PBS LPS }} \frac{\text { TIPE2 }}{\text { PBS LPS }}$

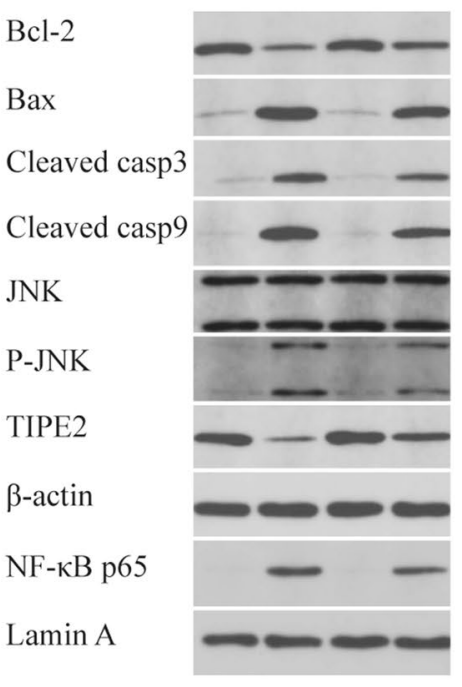

26KD

$21 \mathrm{KD}$

$17 \mathrm{KD}$

49KD

$54 \mathrm{KD}$

$46 \mathrm{KD}$

$54 \mathrm{KD}$

46KD

$21 \mathrm{KD}$

$42 \mathrm{KD}$

$65 \mathrm{KD}$

$76 \mathrm{KD}$
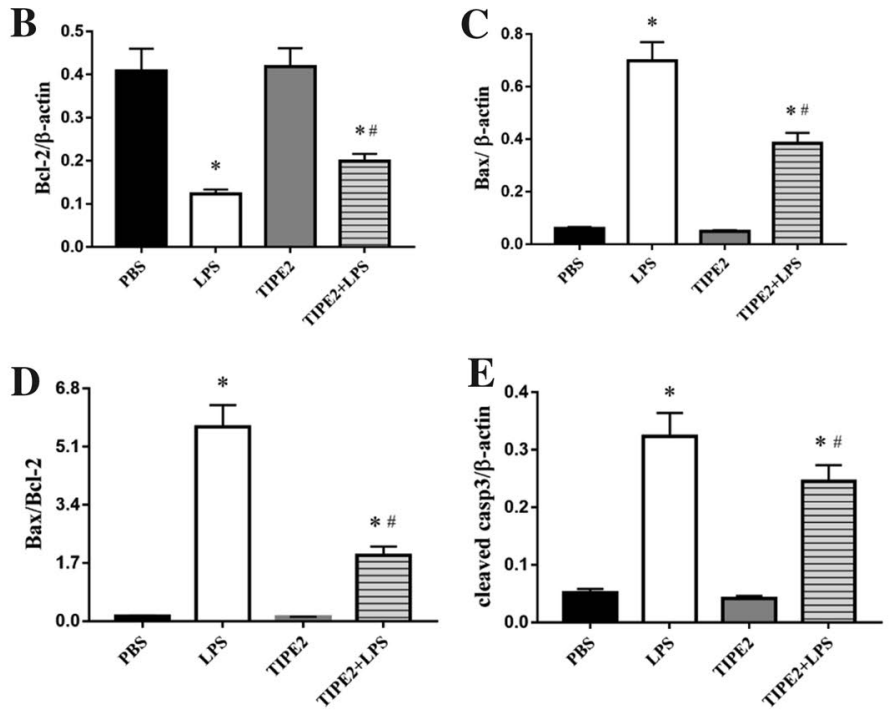
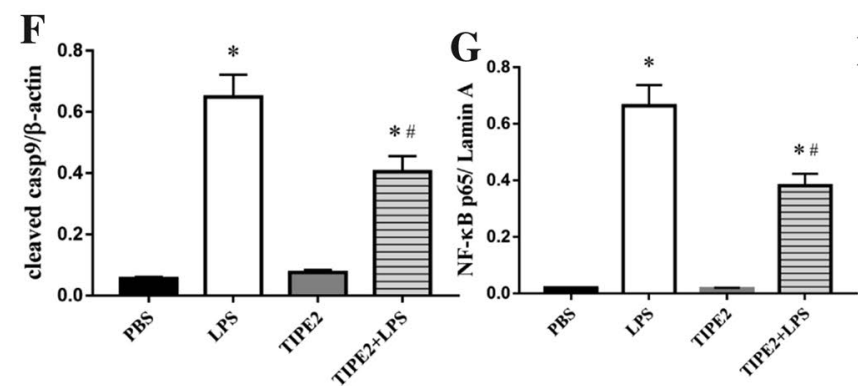

Fig. 7 TIPE2 overexpression suppressed the activation of JNK and $\mathrm{NF}-\mathrm{\kappa B}$ as well as the protein expression of genes involved in apoptosis and lung injury induced by LPS. The mice were treated as described in Fig. 1. Lung tissues were collected for western blotting analysis at $24 \mathrm{~h}$ after LPS or PBS administration. a Western blotting analysis of protein expression of Bax, cleaved caspase-3, cleaved cas-
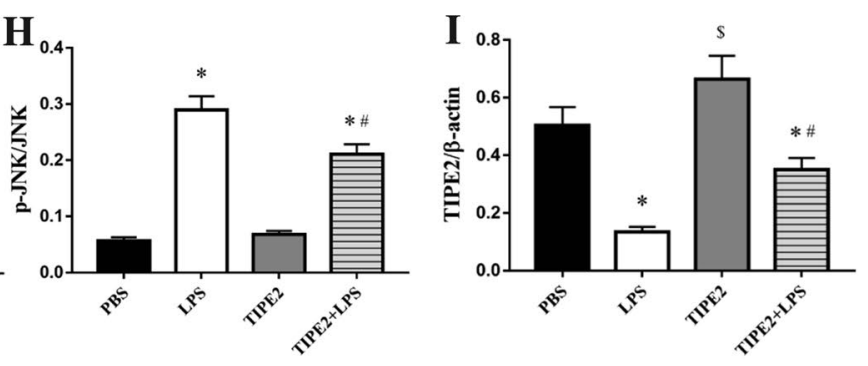

lung inflammation and apoptosis, which may be associated with decreased NF- $\kappa$ B and JNK activity.

The characteristics of ALI/ARDS can be reproduced by the intracerebral administration of LPS [18], which acts via TLR4 to induce the production of inflammatory cytokines, resulting in damage to microvascular and epithelial integrity and increased alveolar and interstitial edema [19]. In this study, we successfully produced a mouse model of ALI by the intratracheal administration of LPS. We found that acute lung injury, which was characterised by pathological changes in lung tissue (by H\&E staining and TEM), increased lung water content, the infiltration of inflammatory cells, and pulmonary dysfunction was present $24 \mathrm{~h}$ after LPS administration. However, AAV-TIPE2 treatment markedly reduced LPS-induced lung injury.

TIPE2 is a critical regulator of immune homeostasis that negatively regulates $\mathrm{T}$ cell receptor (TCR) and Toll-like receptor (TLR) signalling $[15,20]$. TIPE2 deficiency in mice pase-9, Bcl-2, JNK, p-JNK, nuclear NF-кB p65, and TIPE2; b-h The relative ratio of Bax, cleaved caspase-3, cleaved caspase-9, Bcl-2, $\mathrm{p}-\mathrm{JNK}$, nuclear NF-кB p65, and TIPE2 protein expression. The data are presented as mean \pm SEM. $n=10$ /group, ${ }^{*} P<0.05$ versus $\mathrm{PBS}$ group; ${ }^{\#} P<0.05$ versus LPS group

induces foetal inflammatory diseases, and TIPE2 downregulation in humans causes systemic autoimmunity [15, 21]. Moreover, TIPE2 has also been identified as an apoptosis regulator that contains a death effector domain (DED) and is able to inhibit the activities of the apoptotic enzymes caspase- 3 and caspase- 8 [22]. Sun et al. demonstrated that the deletion of TIPE2 amplifies JNK and p38 MAPK phosphorylation and NF- $\mathrm{KB}$ activation, suggesting that TIPE2 is a negative regulator of JNK, p38 MAPK and NF- $\mathrm{KB}$ [15].

In LPS-induced ALI, LPS is recognized by TLR4 and subsequently promotes the activation of NF- $\mathrm{KB}$ [9], which is a pivotal transcription factor in the pathogenesis of ALI. When activated, NF- $\mathrm{KB}$ p 65 translocates to the nucleus, where it triggers the transcription of inflammatory cytokines, such as TNF- $\alpha$, IL-6, and IL-1 $\beta$ [23]. The inactivation of $\mathrm{NF}-\kappa \mathrm{B}$ p65 inhibits inflammation-induced inflammatory cell infiltration, edema, and pro-inflammatory cytokine production in the lungs [24]. In the current study, TIPE2 
Fig. 8 Immunohistochemical staining of TIPE2 protein expression in the lung. The mice were treated as described in Fig. 1. Lung tissues were collected for immunohistochemical staining at $24 \mathrm{~h}$ after LPS or PBS administration. Representative images of lung tissues under light microscope (Immunohistochemical staining, magnification, $\times 200$ )

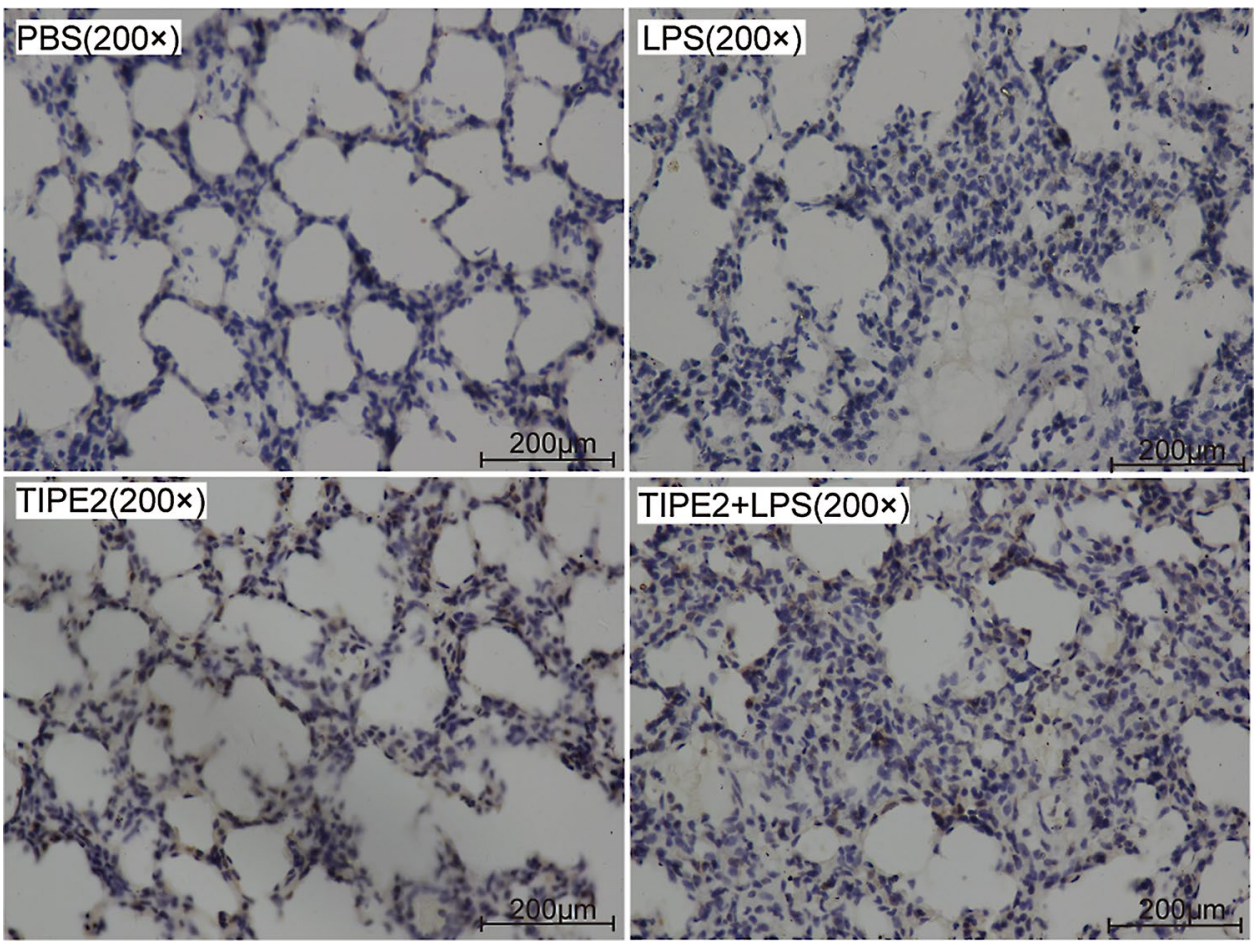

overexpression significantly inhibited the LPS-induced levels of TNF- $\alpha$, IL- 6 , and IL- $1 \beta$ in the serum-induced and concomitantly decreased NF- $\kappa \mathrm{B}$ activation. Therefore, the inhibitory effect of TIPE2 overexpression on LPS-induced increases in the levels of pro-inflammatory cytokines may be ascribed to its suppression of $\mathrm{NF}-\kappa \mathrm{B}$ activation.

Liu MW et al. found that, after LPS challenge, the increased expression of TIPE2 is associated with the inhibition of NF- $\kappa \mathrm{B}$ expression, the production of TNF- $\alpha$ and IL-6 and decreased ROS activity in RAW264.7 cells [25]. In another study by Liu et al., Melilotus extract was shown to have protective effects against CLP-induced lung injury, possibly by upregulating the expression of TIPE2 [26]. Thus, TIPE2 may have a protective effect against sepsis-induced lung injury and LPS challenge in cells. In our present study, AAV-TIPE2 treatment markedly reduced LPS-induced lung injury.

Pulmonary cell apoptosis also plays a critical role in the pathogenesis of ALI [27]. LPS-induced cell apoptosis may be partly dependent on the mitochondria pathway [28]. JNK, a member of the MAPK family, is critical for LPS-induced apoptosis in macrophages [13]. LPS triggers JNK phosphorylation, which subsequently mediates the phosphorylation of anti-apoptotic proteins (Bcl-2/Bcl-xL) and upregulates proapoptotic Bax. When the $\mathrm{Bax} / \mathrm{Bcl}-2$ ratio is elevated, the mitochondrial membrane potential changes, causing the release of cytochrome $\mathrm{C}$ into the cytosol from the mitochondria [29] and resulting in the activation of caspase-9 and then caspase-3 to induce apoptosis [30]. Several previous studies have shown that TIPE2 contains a death effector domain (DED) and that the overexpression of TIPE2 is associated with enhanced survival and the inhibition of caspasemediated apoptosis through the inhibition of the activities of the apoptotic enzymes caspase- 8 and caspase-3 [31-33]. In addition, the depletion of TIPE2 enhances cell death [34, 35]. In the current study, we found that AAV-TIPE2 administration remarkably inhibited the expression of pro-apoptotic proteins (Bax, cleaved caspase-9, and cleaved caspase-3) and JNK phosphorylation and restored the expression of the antiapoptotic protein $\mathrm{Bcl}-2$. These findings suggest that epithelial TIPE2 drives the protective effects against LPS-induced injury and that the inhibitory effect of TIPE2 overexpression on LPS-induced cell apoptosis may be attributed to its suppression of JNK activation.

Together, TIPE2 exerts a protective effect against LPSinduced ALI possibly through its anti-inflammatory and antiapoptotic activities, and it may be a potential therapeutic target for ALI.

In conclusion, our study demonstrated that TIPE2 expression was significantly decreased in lung tissues in ALI mice after LPS challenge. Moreover, adeno-associated virus-mediated TIPE2 overexpression remarkably inhibited inflammation and cell apoptosis induced by LPS. We also provided evidence that the anti-inflammatory and anti-apoptotic effects of TIPE2 might at least partly involve the inhibition of NF- $\mathrm{KB}$ and JNK activation, respectively. Therefore, our results indicate that TIPE2 might be useful as a potential therapeutic target for sepsis-induced ALI. 
Acknowledgements This work was supported by research grants from the National Natural Science Foundation of China (no. 81571941).

\section{Compliance with ethical standards}

Conflict of interest The authors declare no conflict of interest.

\section{References}

1. Ware LB, Matthay MA. The acute respiratory distress syndrome. N Engl J Med. 2000;342(18):1334-49.

2. Li C, Yang D, Cao X, Wang F, Jiang H, Guo H, Du L, Guo Q, Yin X. LFG-500, a newly synthesized flavonoid, attenuates lipopolysaccharide-induced acute lung injury and inflammation in mice. Biochem Pharmacol. 2016;113:57-69.

3. Zhao Z, Tang X, Zhao X, Zhang M, Zhang W, Hou S, et al. Tylvalosin exhibits anti-inflammatory property and attenuates acute lung injury in different models possibly through suppression of NF-kappaB activation. Biochem Pharmacol. 2014;90(1):73-87.

4. Georgene S, George G, Tony TC, Nagamani S. Incidence and outcome of acute lung injury and acute respiratory distress syndrome in the surgical intensive care unit. Indian J Crit Care Med. 2014;18(10):659-65.

5. Zgraggen BR, Tornic J, Müller-Edenborn B, Reyes L, Booy C, Beck-Schimmer B. Acute lung injury: apoptosis in effector and target cells of the upper and lower airway compartment. Clin Exp Immunol. 2010;161(2):324-31.

6. Kawasaki M, Kuwano K, Hagimoto N, Matsuba T, Kunitake R, Tanaka T, et al. Protection from lethal apoptosis in lipopolysaccharide-induced acute lung injury in mice by a caspase inhibitor. Am J Pathol. 2000;157(2):597-603.

7. Wan L, Meng D, Wang H, Wan S, Jiang S, Huang S, et al. Preventive and therapeutic effects of thymol in a lipopolysaccharide-induced acute lung injury mice model. Inflammation. 2018;41(1):183-92.

8. Boonstra A, Rajsbaum R, Holman M, Marques R, Asselin-Paturel C, Pereira JP, et al. Macrophages and myeloid dendritic cells, but not plasmacytoid dendritic cells, produce IL-10 in response to MyD88- and TRIF-dependent TLR signals, and TLR-independent signals. J Immunol. 2006;177(11):7551-8.

9. Pahl HL. Activators and target genes of Rel/NF-kappaB transcription factors. Oncogene. 1999;18(49):6853-66.

10. Cui J, Zhang M, Zhang YQ, Xu ZH. JNK pathway: diseases and therapeutic potential. Acta Pharmacol Sin. 2007;28(5):601-8.

11. Johnson GL, Nakamura K. The c-jun kinase/stress-activated pathway: regulation, function and role in human disease. Biochim Biophys Acta. 2007;1773(8):1341-8.

12. Xu L, Yu J, Zhai DX, Zhang DY, Shen W, Bai LL, et al. Role of JNK activation and mitochondrial Bax translocation in allicininduced apoptosis in human ovarian cancer SKOV3 cells. Evid Based Complement Alternat Med. 2014;2014:378684.

13. Guha M, Mackman N. LPS induction of gene expression in human monocytes. Cell Signal. 2001;13(2):85-94.

14. Kumar D, Gokhale P, Broustas C, Chakravarty D, Ahmad I, Kasid U. Expression of SCC-S2, an antiapoptotic molecule, correlates with enhanced proliferation and tumorigenicity of MDA-MB 435 cells. Oncogene. 2004;23(2):612-6.

15. Sun H, Gong S, Carmody RJ, Hilliard A, Li L, Sun J, et al. TIPE2, a negative regulator of innate and adaptive immunity that maintains immune homeostasis. Cell. 2008;133(3):415-26.

16. Li B, Zeng M, He W, Huang X, Luo L, Zhang H, et al. Ghrelin protects alveolar macrophages against lipopolysaccharideinduced apoptosis through growth hormone secretagogue receptor 1a-dependent c-Jun $\mathrm{N}$-terminal kinase and Wnt/betacatenin signaling and suppresses lung inflammation. Endocrinology. 2015;156(1):203-17.

17. Matute-Bello G, Frevert CW, Martin TR. Animal models of acute lung injury. Am J Physiol Lung Cell Mol Physiol. 2008;295(3):L379-99.

18. Su X, Wang L, Song Y, Bai C. Inhibition of inflammatory responses by ambroxol, a mucolytic agent, in a murine model of acute lung injury induced by lipopolysaccharide. Intensive Care Med. 2004;30(1):133-40.

19. Johnson Elizabeth R, Matthay Michael A. Acute lung injury: epidemiology, pathogenesis, and treatment. J Aerosol Med Pulm Drug Deliv. 2010;23(4):243-52.

20. Chen H, Bai C, Wang X. The value of the lipopolysaccharideinduced acute lung injury model in respiratory medicine. Expert Rev Respir Med. 2010;4(6):773-83.

21. Freundt EC, Bidere N, Lenardo MJ. A different TIPE of immune homeostasis. Cell. 2008;133(3):401-2.

22. Li D, Song L, Fan Y, Li X, Li Y, Chen J, et al. Down-regulation of TIPE2 mRNA expression in peripheral blood mononuclear cells from patients with systemic lupus erythematosus. Clin Immunol. 2009;133(3):422-7.

23. Kumar D, Whiteside TL, Kasid U. Identification of a novel tumor necrosis factor-alpha-inducible gene, SCC-S2, containing the consensus sequence of a death effector domain of fasassociated death domain-like interleukin- 1beta-converting enzyme-inhibitory protein. J Biol Chem. 2000;275(4):2973-8.

24. Wang H, Wang L, Li N, Li J, Yu F, Zhao Y, et al. Subanesthetic isoflurane reduces zymosan-induced inflammation in murine kupffer cells by inhibiting ROS-activated p38 MAPK/NF- $\kappa \mathrm{B}$ signaling. Oxid Med Cell Longev. 2014;2014:851692.

25. Liu MW, Su MX, Zhang W, Qian CY. Atorvastatin increases lipopolysaccharide-induced expression of tumour necrosis factor- $\alpha$-induced protein 8 -like 2 in RAW264.7 cells. Exp Ther Med. 2014;8(1):219-28.

26. Liu MW, Su MX, Wang YH, Qian CY. Effect of Melilotus extract on lung injury via the upregulation of tumor necrosis factor- $\alpha$-induced protein-8-like 2 in septic mice. Mol Med Rep. 2015;11(3):1675-84.

27. Blackwell TS, Blackwell TR, Holden EP, Christman BW, Christman JW. In vivo antioxidant treatment suppresses nuclear factor-kappa B activation and neutrophilic lung inflammation. J Immunol. 1996;157(4):1630-7.

28. Galani V, Tatsaki E, Bai M, Kitsoulis P, Lekka M, Nakos G, et al. The role of apoptosis in the pathophysiology of Acute Respiratory Distress Syndrome (ARDS): an up-to-date cell-specific review. Pathol Res Pract. 2010;206(3):145-50.

29. Aggarwal S, Dimitropoulou C, Lu Q, Black SM, Sharma S. Glutathione supplementation attenuates lipopolysaccharide-induced mitochondrial dysfunction and apoptosis in a mouse model of acute lung injury. Front Physiol. 2012;3:161.

30. Kluck RM, Bossy-Wetzel E, Green DR, Newmeyer DD. The release of cytochrome $\mathrm{c}$ from mitochondria: a primary site for Bcl-2 regulation of apoptosis. Science. 1997;275(5303):1132-6.

31. Kumar D, Whiteside TL, Kasid U. Identification of a novel tumor necrosis factor-alpha-inducible gene, SCC-S2, containing the consensus sequence of a death effector domain of fas-associated death domain-like interleukin-1beta-converting enzyme-inhibitory protein. J Biol Chem. 2000;275:2973-8.

32. You Z, Ouyang H, Lopatin D, Polver PJ, Wang CY. Nuclear factorkappa B-inducible death effector domain-containing protein suppresses tumor necrosis factor-mediated apoptosis by inhibiting caspase-8 activity. J Biol Chem. 2001;276:26398-404. 
33. Kumar D, Gokhale P, Broustas C, Chakravarty D, Ahmad I, Kasid U. Expression of SCC-S2, an antiapoptotic molecule, correlates with enhanced proliferation and tumorigenicity of MDA-MB 435 cells. Oncogene. 2004;23:612-6.

34. Zhang HG, Hyde K, Page GP, Brand JP, Zhou J, Yu S, et al. Novel tumor necrosis factor alpha-regulated genes in rheumatoid arthritis. Arthritis Rheum. 2004;50(2):420-31.
35. Zhang C, Chakravarty D, Sakabe I, Mewani RR, Boudreau HE, Kumar D, et al. Role of SCC-S2 in experimental metastasis and modulation of VEGFR-2, MMP-1, and MMP-9 expression. Mol Ther. 2006;13(5):947-55.

Publisher's Note Springer Nature remains neutral with regard to jurisdictional claims in published maps and institutional affiliations. 